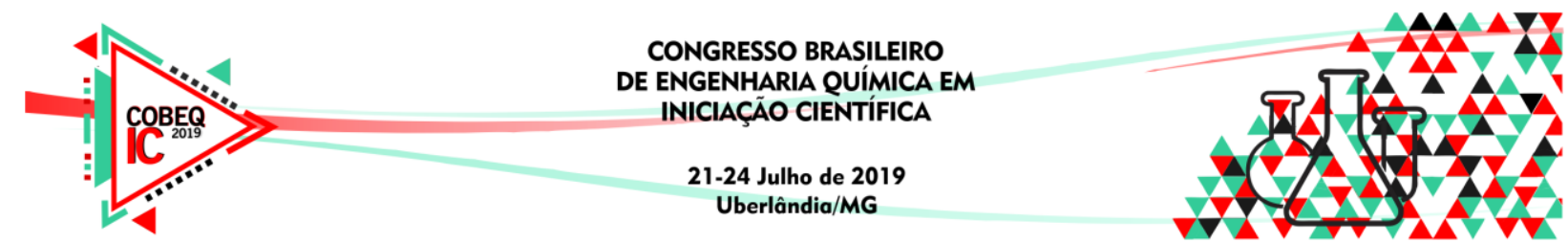

\title{
USO DE RESÍDUOS TRATADOS PARA ADSORÇÃO DO CORANTE AZUL BRILHANTE REMAZOL EM EFLUENTE SINTÉTICO
}

\author{
J. P. S. FERNANDES ${ }^{1}$, A. L. A. CORREA ${ }^{2}$, L. B. PIMENTA ${ }^{2}$, J. T. CLARO ${ }^{2}$, C. A. F. \\ PEREIRA $^{3}$
}

${ }^{1}$ Instituto Federal do Norte de Minas Gerais, Departamento de Engenharia Química

${ }^{2}$ Faculdade de Ciência e Tecnologia de Montes Claros, Faculdade de Engenharia Química

${ }^{3}$ Universidade Federal dos Vales do Jequitinhonha e Mucuri, Departamento de Engenharia

Química

E-mail para contato:.jpsf@aluno.ifnmg.edu.br

\begin{abstract}
RESUMO - A conservação ambiental e a reutilização de insumos têm sido temas de grande discussão em relação às indústrias químicas. Diversos trabalhos sugerem o uso de resíduos abundantes, de matriz porosa, na adsorção de contaminantes químicos. Esse trabalho buscou comparar o potencial de adsorção dos adsorventes sílica, lodo e bagaços de cana e de malte como formas de remover o corante azul brilhante remazol em efluente sintético. Avaliou-se a influência da massa e as isotermas de adsorção pelo modelo de Freundlich. O bagaço de cana foi o adsorvente que mais sofreu variação com o aumento da massa, enquanto o de malte teve a melhor adsorção na maior massa utilizada. Pelos resultados dos parâmetros de Freundlich, verificou-se que apesar de tratamentos físico-químicos o lodo e a sílica não apresentaram melhores desempenhos. Os bagaços de cana e de malte tiveram isotermas favoráveis por possuírem superfícies heterogêneas e disformes fazendo com os sítios não fossem ocupados da mesma forma. Os bagaços in natura, também, tiveram maiores constantes de Freundlich e, portanto, maiores energias médias de adsorção, enquanto os demais tiveram valores insignificantes.
\end{abstract}

\section{INTRODUÇÃO}

A indústria e o governo tornaram mais conscientes da necessidade de tratar os efluentes industriais e reduzir a poluição dos rios. As normas para a qualidade de águas residuais estão gradualmente se tornando mais rígidas e novos tratamentos estão sendo continuamente investigados e desenvolvidos. Numerosos tipos de materiais residuais são usados em métodos padrões para tratamento de água (Poots, McKay \& Healy, 1976).

Embora o carvão ativado remova os corantes da solução, eles continuam presentes em uma forma mais concentrada e tóxica, e assim sua eliminação segura aumenta ainda mais os custos. Portanto, existe potencial para que o processo de remoção de corante por adsorção seja mais viável economicamente observando o uso de biossorventes de menor custo (Robinson, Chandran \& Nigam, 2002). Sendo assim, o presente trabalho teve por principal objetivo comparar materiais residuais para adsorção de corante. 


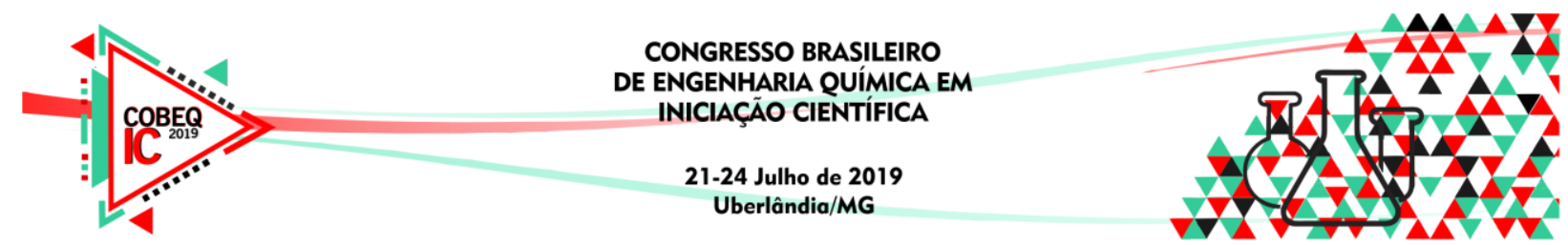

\section{METODOLOGIA}

a) Pré-tratamento dos resíduos

Para ambos os bagaços (Malte e Cana) realizou-se os mesmos procedimentos. Sendo assim, houve a remoção das impurezas através de água corrente e mili-Q. Para secagem, os materiais foram submetidos à secagem por 24 horas em temperatura de $105^{\circ} \mathrm{C}$. Posteriormente, fez-se a pulverização em liquidificador industrial para aumento da superfície de contato (Gaebrin, 2014).

Conforme metodologia descrita por Pereira et al. (2018), na obtenção da sílica realizouse lavagem das cascas de arroz com ácido clorídrico a $10 \%$ numa proporção de 1:9 m/v. Em seguida, o material foi levado a uma autoclave à temperatura de $121^{\circ} \mathrm{C}$ e $1,5 \mathrm{kgf} \mathrm{cm}^{-2} \mathrm{de}$ pressão em 1 hora. Depois, fez-se outra lavagem com água mili-Q até neutralização para secagem em estufa. Por último, as cascas tratadas foram calcinadas a $650^{\circ} \mathrm{C}$ em 4 horas no forno mufla.

Uma mistura de proporção de $2: 1 \mathrm{~m} / \mathrm{m}$ de lodo e hidróxido de potássio $(\mathrm{KOH})$ foi colocada na mufla a $550^{\circ} \mathrm{C}$ durante 45 minutos. Obtido o carvão procedeu-se para a sua ativação com a lavagem a partir de ácido clorídrico $(\mathrm{HCl})$ usando-se $18,75 \mathrm{~mL}$ para cada grama da cinza obtida. A mistura permaneceu à $27^{\circ} \mathrm{C}$ sob agitação constante por cerca de 2 horas e em seguida foi filtrada com água quente à $90^{\circ} \mathrm{C}$ e acondicionada na estufa à $150^{\circ} \mathrm{C}$ por 24 horas (Lopes, 2016).

\section{b) Construção da curva analítica}

A construção da curva padrão com a absorbância em função de diferentes concentrações da solução de corante é de fundamental importância para o trabalho, uma vez que ela permite as determinações das concentrações de equilíbrio, com isso, a partir de soluções de até 200 $\mathrm{mg} \mathrm{L}^{-1}$ do efluente sintético fez-se a leitura em espectrofotômetro da absorbância (modelo NOVA 1600UV com faixa de 190-1100 nm). O comprimento utilizado foi de $580 \mathrm{~nm}$ (SILVA, 2017).

\section{c) Influência da massa}

Conforme mostrado por Zanutto \& Silva (2016), a relação entre a massa de adsorvente e a quantidade de corante removido é importante para que se estude a viabilidade do processo de adsorção. Dito isso, o estudo da influência da massa foi feito na faixa de 0,2 a 2 gramas e volume fixo de $20 \mathrm{~mL}$ de efluente sintético nas mesmas condições operacionais: rotação = $200 \mathrm{rpm} ;$ Tempo $=1 \mathrm{~h}$; Temperatura $=30^{\circ} \mathrm{C} ; \mathrm{pH}=7,3 ; \mathrm{C}=200 \mathrm{mg} \mathrm{L}^{-1}$.

\section{d) Isotermas de Adsorção}

$\mathrm{O}$ ensaio de isoterma foi realizado em um shaker a $200 \mathrm{rpm}$ e $30^{\circ} \mathrm{C}$ por 1 hora. Utilizou-se soluções de 40 a $200 \mathrm{mg} \mathrm{L}^{-1}$, preparadas a partir de uma solução padrão de 500 $\mathrm{mg} \mathrm{L}^{-1}$ do corante azul brilhante remazol. Ao final do processo, as amostras foram filtradas e tiveram as absorbâncias (concentrações de equilíbrio) lidas em espectrofotômetro. A partir do modelo de Freundlich e os dados de capacidade de adsorção, linearizou-se para obtenção dos parâmetros da Equação 1.

$$
\log q_{e}=\log K_{f}+\frac{1}{n} \log C_{e}
$$




\section{RESULTADOS}

Figura 1 - (a) Curva padrão do corante; e, (b) Influência da massa

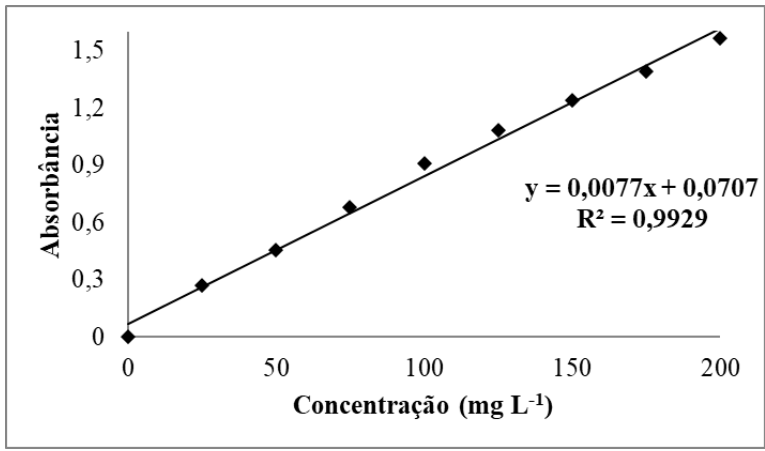

(a)

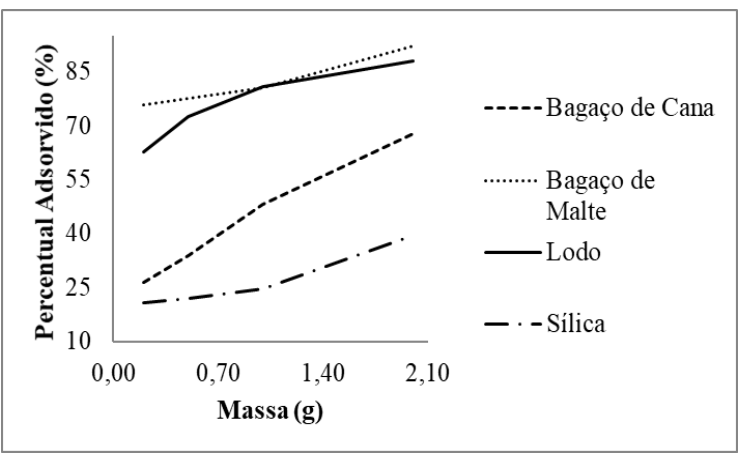

(b)

Figura 2 - Isoterma (Modelo de Freundlich): (a) Lodo ativado; e, (b) Sílica

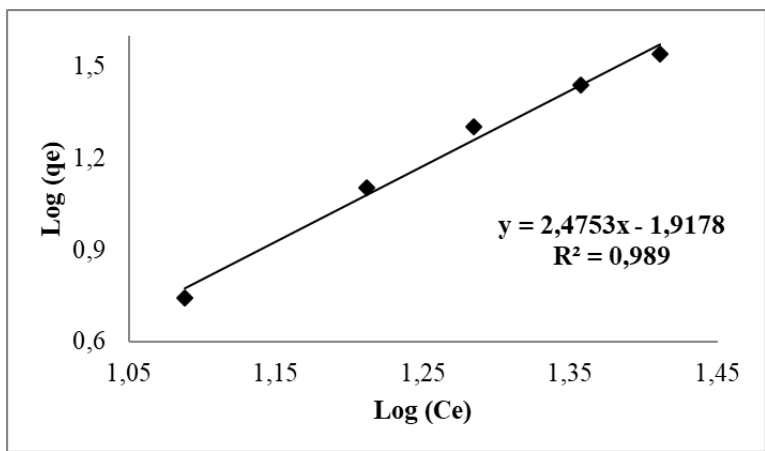

(a)

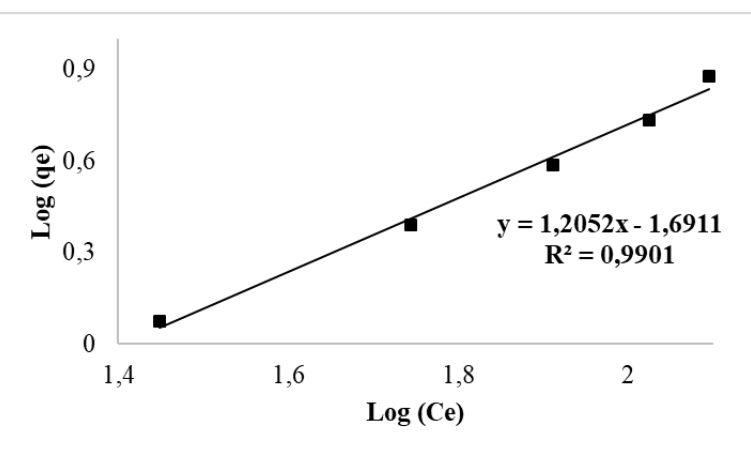

(b)

Figura 3 - Isoterma (Modelo de Freundlich): (a) Bagaço de Malte; e, (b) Bagaço de Cana

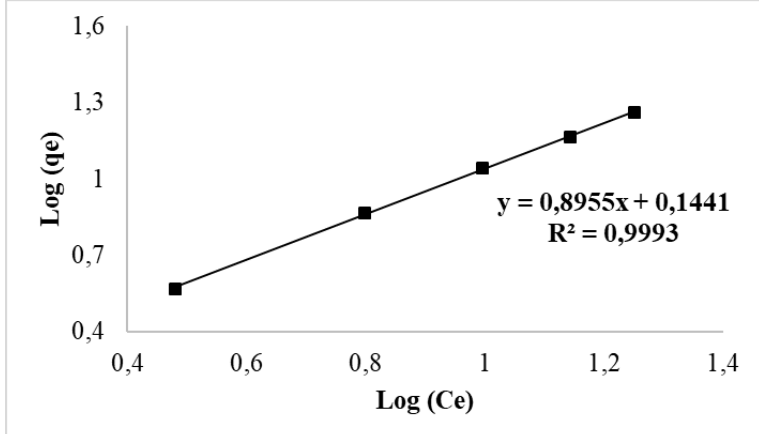

(a)

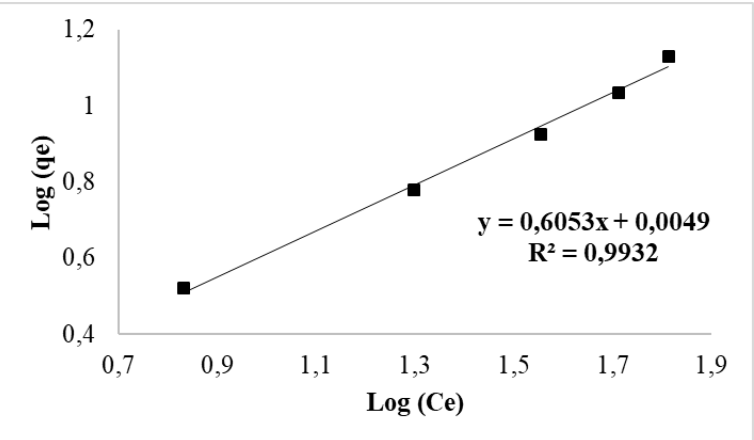

(b)

Tabela 1 - Parâmetros do modelo de Freundlich para os 4 (quatro) adsorventes

\begin{tabular}{cccc}
\hline Adsorvente & $\mathbf{K}_{\mathbf{F}}\left(\mathbf{m g}^{\mathbf{1 - ( 1 / \mathbf { n } )}}\left(\mathbf{g}^{\mathbf{- 1}}\right) \mathbf{L}^{\mathbf{1 / n}}\right)$ & $\mathbf{1 / n}$ & $\mathbf{n}$ \\
\hline Lodo & $0,012 \pm 0,001$ & $2,4753 \pm 0,052$ & $0,403 \pm 0,003$ \\
Sílica & $0,020 \pm 0,003$ & $1,2052 \pm 0,033$ & $0,829 \pm 0,001$ \\
Bagaço de Malte & $1,393 \pm 0,041$ & $0,8955 \pm 0,004$ & $1,116 \pm 0,020$ \\
Bagaço de Cana & $1,011 \pm 0,034$ & $0,6053 \pm 0,001$ & $1,652 \pm 0,014$ \\
\hline
\end{tabular}




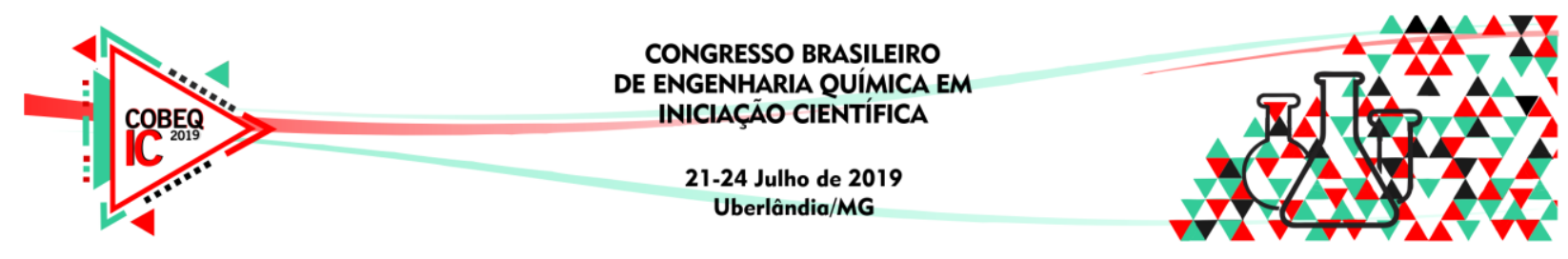

\section{DISCUSSÃO}

Pela Figura 1a observa-se a curva padrão construída para o efluente sintético, em que pelo coeficiente de correlação verifica-se que $99,6 \%$ das variações da absorbância podem ser explicadas pela concentração. A absortividade molar encontrada foi de $0.0077 \mathrm{~L} \mathrm{mg}^{-1} \mathrm{~min}^{-1}$. Já atentando-se a influência da massa representada em $1 \mathrm{~b}$, verificou-se que o bagaço de malte foi o adsorvente que apresentou melhor capacidade adsortiva para todas as quantidades de massa, porém, apresentou pouca variação com o aumento. Para quantidades de adsorventes próximas a $1 \mathrm{~g}$ os bagaços se comportaram da mesma foram. O lodo e a sílica tiveram variação razoável com o aumento da massa. Mesmo com o tratamento físico-químico as cinzas da casca de arroz apresentaram o pior desempenho.

Nas Figuras 2, 3 e 4 para o modelo de Freundlich (equação 1), todas as regressões apresentaram boas correlações $(r \geq 0,994)$, dessa forma, os parâmetros obtidos são passíveis de análises conforme a literatura. Pela Tabela 1, verifica-se que das constantes de Freundlich $\left(\mathbf{K}_{\mathbf{F}}\right)$, as dos bagaços de malte e de cana apresentaram valor mais expressivo, sendo assim, para esses adsorventes as energias médias de adsorção foram maiores, já a do lodo e da sílica foram quase insignificantes. Quanto ao parâmetro 1/n verifica-se que o valor para a sílica ficou próximo a 1 mostrando a tendência para tipo de isoterma linear enquanto para o lodo o valor foi maior que 1 mostrando maior atração do solvente pelo adsorvente. Por último, o parâmetro n mostra que os bagaços de malte e de cana apresentam isotermas favoráveis $(1<\mathrm{n}<10)$ (Nascimento et al., 2014; Cooney, 1999; Delle-Site, 2001).

\section{CONCLUSÃO}

A partir da metodologia empregada e dos resultados obtidos, verifica-se que os adsorventes usados constituem alternativas para adsorção do corante azul brilhante remazol. O objetivo da pesquisa foi alcançado ao verificar, em comparação, que mesmo realizando tratamentos físico-químicos para a sílica e o lodo, os bagaços de cana e de malte in natura apresentaram melhores resultados devido às superfícies heterogêneas.

\section{NOMENCLATURA}

$$
\begin{aligned}
& \text { qe - quantidade de soluto adsorvido }\left(\mathrm{mg} \mathrm{g}^{-1}\right) ; \\
& \mathbf{C e} \text { - concentração de equilíbrio em solução }\left(\mathrm{mg} \mathrm{L}^{-1}\right) ; \\
& \mathbf{1} / \mathbf{n} \text { - constante relacionada à heterogeneidade da superfície }(-) ; \\
& \mathbf{K}_{\mathbf{F}} \text { - constante de capacidade de adsorção de Freundlich }\left(\mathrm{mg}^{1-(1 / \mathrm{n})}\left(\mathrm{g}^{-1}\right) \mathrm{L}^{1 / \mathrm{n}}\right) \text {. }
\end{aligned}
$$

\section{REFERÊNCIAS}

COONEY, D. O. Adsorption Design for Wastewater Treatment. Florida: CRC Press, 1999. 


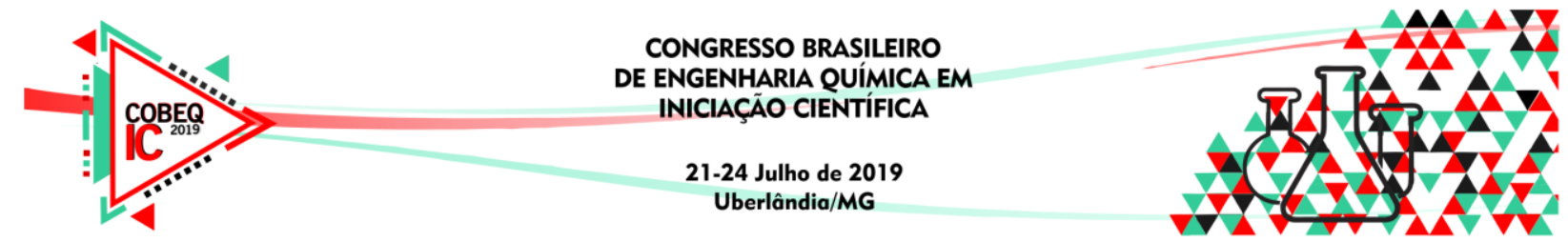

DELLE-SITE, A. Factors affecting sorption of organic compounds in natural sor-bent/water systems and sorption coefficients for selected pollutants. A review. Journal of Physical and Chemical Reference Data, v. 30, p. 187-439, 2001. Disponível em: <http://www.nist.gov/data/PDFfiles/jpcrd598.pdf>. Acesso em: 10 abr. 2019.

GAEBRIN, G. O. Secagem do bagaço de cana-de-açúcar e estudo da sua aplicação na remoção de metal pesado. Paraná: Toledo, 2014. [Dissertação do Programa de PósGraduação em Engenharia Química da UNIOESTE];

NASCIMENTO, R. F.; LIMA, A. C. A.; VIDAL, C. B.; MELO, D. Q.; RAULINO, G. S. C.; Adsorção: aspectos teóricos e aplicações ambientais, 1a. Ed., Imprensa Universitária: Fortaleza-Ceará, 2014.

PEREIRA, C. A. P.; MACEDO, A. L.; FIDENCIO, P. H.; FABRIS, J. D.; FERNANDES, J. P. S. Síntese de catalisador heterogêneo a partir da sílica extraída da casca de arroz e iodeto de potássio para a aplicação em Tereações de produção de biodiesel. Maranhão: São Luís, 2018. Anais do $58^{\circ}$ Congresso Brasileiro de Química;

POOTS, V. J. P.; MCKAY, G.; HEALY, J. J. The removal of acid dye from effluent using natural adsorbents - I peat, Water Research, Volume 10, 1976, 1061-1066.

ROBINSON, T.; CHANDRAN, B.; NIGAM, P. Removal of dyes from a synthetic textile dye effluent by biosorption on apple pomace and wheat straw, Water Research, 2002, 28242830 .

SILVA, A. B. C.; ANDRADE, R. M. F.; FREIRE, F. B.; NAGALLI, A.; CARVALHO, K. Q.; PASSIG, F. H.; KREUTZ, C. Análise da utilização de cerâmica vermelha como adsorvente na remoção do corante têxtil Direct Blue de uma solução aquosa, Revista Matéria, v. 22, n. 3, 2017;

ZANUTTO, A.; SILVA, B. C. Avaliação do bagaço de malte como biossorvente do corante amarelo Reafix B2R. Paraná: Ponta Grossa, 2016. [Trabalho de Conclusão do Curso de Engenharia Química da UFTPR]; 